nuestra Sociedad deberá ser un líder opinante en esos cambios.

Creo que nuestro Directorio en particular, debe responsabilizarse de algunas tareas especificas: la realización de la FIGO 2003 y nuestro posicionamiento en ese organismo exige que hagamos lo posible por mantener un nexo internacional importante. La incorporación de especialistas de provincia y el trabajo conjunto con los de países latinoamericanos es hoy una exigencia para el futuro. El realizar el Congreso Chileno fuera de Santiago y realizar jornadas en el norte o sur del país, me parece también una exigencia ineludible; realizar algunas modificaciones de nuestras reuniones periódicas, de modo de favorecer una asistencia mayor que la pobre asistencia actual, también me parece fundamental. Quizás podemos hacer reuniones de consenso similares a las mesas redondas de años atrás, y quizás, debemos pensar en entregar a diferentes hospitales la responsabilidad de algunas reuniones; el quehacer de la Sociedad debe aunar a las múltiples sociedades satélites que hoy existen; la elección de los directorios de la Sociedad probablemente también debe modificarse, de modo de que un comité de búsqueda integrado por past presidents y otros integrantes distinguidos de la Sociedad sean los que oficialmente busquen, entrevistando a especialistas de diferentes hospitales, a quienes deben dirigir los destinos de la Sociedad en el futuro.

La supervivencia de nuestra Sociedad incluye la revista propia y la realización de un Congreso cada dos años, pero esto está lejos de ser todo. Nuestra Sociedad debe asumir su rol como organismo autónomo, consultor y técnico; debe ser un líder de opinión en lo que a nuestras materias atañe.

Es claro que las tareas son muchas y de gran envergadura. Para su ejecución, un directorio o sus miembros no bastan, y debemos delegar encargos específicos a muchos de ustedes. Las tareas de este o de cualquier grupo no son de unos pocos individuos, sino de todos los que conformamos esta Sociedad. Lo que el futuro nos depare será como siempre nuestra responsabilidad y ella es colectiva en lo que a estas materias se refiere. Lo que les pido hoy, entonces, es que soñemos juntos los cambios que queramos realizar, y que cada vez que alcemos la voz para criticar algo que pudimos hacer mejor, pensemos primero en como podemos colaborar para que la próxima vez los resultados sean mejores.

Martes 6 de Abril de 2004.

Dr. EnRIQUe OyARZún Ebensperger

\title{
CUENTA DEL PRESIDENTE DE LA SOCIEDAD CHILENA DE OBSTEIRICIA Y GINECOLOGÍA
}

\section{Estimados Consocios:}

De acuerdo a los Estatutos, me corresponde hoy en mi última actividad oficial como Presidente de la Sociedad Chilena de Obstetricia y Ginecología, dar cuenta de la gestión del directorio que he tenido el honor de presidir durante los años 2002 y 2003. La cuenta financiera debería ser presentada por el Sr. Tesorero Dr. Patricio Silva Rojas, quien por razones de fuerza mayor se ha disculpado de su inasistencia y por lo tanto será entregada por el Dr. Eghon Guzmán Bustamante, Secretario General.

REUNIONES DE DIRECTORIO. Durante los años 2002 y 2003 se realizaron 40 reuniones de
Directorio en las que se trataron varios temas relacionado con la marcha de la Sociedad y principalmente nos abocamos a los Cursos precongreso y a incentivar la inscripción de médicos chilenos y extranjeros al Congreso Mundial FIGO 2003.

REUNIONES CIENTIFICAS. Durante el año 2002 se realizaron 16 sesiones científicas en las que se presentaron 28 trabajos, 10 de ellos fueron trabajos de ingreso a la Sociedad de los siguientes médicos:

1. Dr. Jorge Varela P. "CONIZACIÓN POR ASA. Experiencia en el hospital Carlos Van Buren". Servicio de Obstetricia y Ginecología. Hospital Carlos Van Buren de Valparaíso. 
2. Dr. Alfredo Germain A. "Parto prematuro ASOCIADO A COLESTASIA INTRAHEPÁTICA DEL EMBARAZO. ROL DEL ÁCIDO CÓLICO EN LA MODULACIÓN DE LA VÍA OCITOCINA-RECEPTOR DE OT EN EL MIOMETRIO". Departamentos de Obstetricia y Ginecología, Nefrología y Gastroenterología. Centro de Investigaciones Médicas. Facultad de Medicina. Pontificia Universidad Católica de Chile.

3. Dr. Fernando Abarzúa C. "Prevalencia DE COLONIZACIÓN POR STREPTOCOCCUS AGALACTIAE (GRUPO B) EN EL TERCER TRIMESTRE DEL EMBARAZO. EVALUACIÓN DEL CULTIVO SELECTIVO EN 2192 PACIENTES". Departamento de Obstetricia y Ginecología. UDA Laboratorios Clínicos. Facultad de Medicina. Pontificia Universidad Católica de Chile.

4. Dr. Rodrigo Parada C. "Histerectomía VAGINAL Y CORRECCIÓN DE INCONTINENCIA URINARIA CON TVT (TENSIÓN-FREE VAGINAL TAPE)". Servicios de Obstetricia y Ginecología y de Urología. Hospital Carlos Van Buren de Valparaíso.

5. Dr. Francisco Bueno N. "Tratamiento MÉdICO DEL EMBARAZO ECTÓPICo" Servicio de Obstetricia y Ginecología y de Neonatología. Hospital Clínico San Borja Arriarán. Departamento de Obstetricia y Ginecología Central, Facultad de Medicina, Universidad de Chile.

6. Dr. Horacio Figueroa D. "EFECTO IN VITRO DE LA INSULINA SOBRE LA CAPACIDAD CONTRÁCTIL UTERINA INDUCIDA POR OXITOCINA". Servicio de Obstetricia y Ginecología, Hospital Parroquial de San Bernardo. Laboratorio de Fisiología Molecular y Celular, Facultad de Medicina, Universidad de Los Andes.

7. Dr. Luis Alfonso Fuentes L. "Histeroscopia quirúrgica. Experiencia inicial en el Hospital Claudio Vicuña de San Antonio". Servicio de Obstetricia y Ginecología, Hospital "Claudio Vicuña" de San Antonio.

8. Dra. Pilar Gazmuri P. "Mamografía full DIGITAL O EN CAMPO COMPLETO. EXPERIENCIA CLÍNICARADIOLÓGICA-HISTOLÓGICA". Centro de Diagnóstico Mamario, Laboratorio Citolab.

9. Dr. José Luis Troncoso J. "Tratamiento MÉdICO DEL EMBARAZO ECTÓPICO: EVALUACIÓN PROSPECTIVA DE LA FERTILIDAD". INTEGRAMEDICA Ginecología, Instituto de Investigaciones Materno-Infantil (IDIMI). Departamento y Servicio de Obstetricia y Ginecología, Hospital San Borja-Arriarán, Universidad de Chile.

10. Dr. Masami Yamamoto C. "Rotura PREMAtURA DE MEMBRANAS AL TÉRMINO: MANEJO EXPECTANTE POR 24 HORAS E INDUCCIÓN CON OXITOCINA". Sección de Embarazo Patológico, Unidad de Gestión Clínica de la Mujer y Recién Nacido, Hospital Padre Hurtado.
Una Mesa Redonda el 6 de Agosto. "WHI: RIESGOS DE CÁNCER MAMARIO Y CARDIOVASCULAR CON THR COMBINADA CONTINUA".

Durante el año 2003 se realizaron 14 Sesiones Científicas en las que se presentaron 28 trabajos científicos, 8 de ellos fueron trabajos de ingreso a la Sociedad de los siguientes médicos:

1. Dr. Jorge Figueroa P. "ISOINMUNIZACION RH: EVALUACIÓN DE LA UTILIDAD DEL DOPPLER DE ARTERIA CEREBRAL MEDIA EN LA PREDICIÓN Y MANEJO DE LA ANEMIA FETAL". Servicio y Departamento de Obstetricia y Ginecología, Hospital Clínico San BorjaArriarán.

2. Dr. Andrés Cubillos G. "EFICACIA PROFILÁCTICA DE LA ASOCIACIÓN TINIDAZOL- MICONAZOL EN PACIENTES SOMETIDAS A HISTERECTOMÍA VAGINAL". Servicio de Obstetricia y Ginecología, Hospital Carlos Van Buren de Valparaíso.

3. Dr. Fernando Zegers H. "MultigestacióN COMO MARCADOR DE EFICIENCIA REPRODUCTIVA. ENSEÑANZAS DE LA REPRODUCCIÓN ASISTIDA". Unidad de Medicina Reproductiva y Unidad de Medicina Perinatal, Clínica Las Condes.

4. Dr. Ricardo Pommer T. "Criopreservación DE PRONUCLEOS. ROL EN EL PROGRAMA DE FERTILIZACIÓN ASISTIDA". Servicio y Departamento de Ginecología y Obstetricia, Hospital Clínico San BorjaArriarán, Universidad de Chile.

5. Dr. Pablo Muñoz M. "EXPeriencia y MANEJo DEL PÚRPURA TROMBOCITOPENICO IDIOPÁTICO DURANTE EL EMBARAZO". Servicio de Obstetricia y Ginecología y de Hematología, Hospital Barros Luco Trudeau.

6. Dr. Víctor Quiroz G. "CORDOCENTESIS: EXPERIENCIA LOCAL". Departamento y Servicio de Obstetricia y Ginecología, Hospital Guillermo Grant B, Universidad de Concepción.

7. Dr. Eduardo Faundez P. "CINTA VAGINAL LIBRE DE TENSIÓN EN EL TRATAMIENTO DE LA INCONTINENCIA DE ORINA FEMENINA. UNA NUEVA FORMA DE INSERCIÓN A TRAVÉS DEL AGUJERO OBTURADOR". Servicio de Obstetricia y Ginecología, Hospital Luis Tisné.

8. Dr. Ricardo San Martín T. "CÁNCER CERVICOUTERINO EN ESTADIO IB2: TRATAMIENTO CON RADIOQUIMIOTERAPIA CONCOMITANTE Y CIRUGÍA". Servicio y Departamento de Obstetricia y Ginecología, Unidad de Ginecología Oncológica, Hospital Clínico San Borja Arriarán. Universidad de Chile. Instituto de Radiomedicina (IRAM).

\section{PREMIOS AÑO 2001}

En Santiago de Chile, con fecha 8 de Julio de 2002, a las 12:30 horas, se reúne la Comisión de Premios formada de acuerdo a los artículos 26, 27 
y 29 de los Estatutos de la Sociedad Chilena de Obstetricia y Ginecología. El Jurado constituido por el Presidente de la Sociedad, Dra. Mercedes Ruiz Flores, por los miembros de elección Dr. Ramón Rubio M. y Gabriel Leal C. y por el Secretario General Dr. Eghon Guzmán B., éste último solo con derecho a voz, acuerdan otorgar los siguientes premios correspondientes al año 2001.

1. Premio "DR. JORGE DÍAZ BRAVO", año 2001, al mejor trabajo de ingreso: RESTRICCIÓN DE CRECIMIENTO FETAL: UN HALLAZGO FRECUENTE ENTRE LOS RECIÉN NACIDOS PREMATUROS" de los Drs. Jorge Carvajal C., Yanko Morales S., Alfredo Germain A., del Departamento de Obstetricia y Ginecología, Laboratorio de Medicina Materno Fetal, Pontificia Universidad Católica de Chile. El Jurado acordó otorgar una "Mención Honrosa" del premio "Dr. Jorge Díaz Bravo", año 2001, al trabajo: EVALUACIÓN DE LA HISTERECTOMÍA VAGINAL EN AUSENCIA DE PROLAPSO" de los Drs. Christian Franck K., Paola Castro C., Erasmo Bravo O., Carlos Bennett R., Julián Prado G., Alejandro Carmona B., Servicio de Obstetricia y Ginecología, Unidad de Ginecología, Hospital Carlos Van Buren.

2. Premio "DR. JUAN MALFANTI", Año 2001, al mejor trabajo de casuística, "MELANOMA MALIGNO EN UN QUISTE DERMOIDE DE OVARIO" de los Drs. Santiago Parry R., Christian Franck K., del Servicio de Ginecología Hospital Carlos Van Buren de Valparaíso.

3. Premio "DR. JUAN WOOD WALTERS", año 2001, al mejor trabajo de Ginecología, "ANTIGENO DE CARCINOMA EPIDERMOIDE (SCC) COMO MARCADOR TUMORAL EN CANCER CERVICOUTERINO" de los Drs. Roberto Yazigi I., Rodrigo Castillo B., Giuseppe Aliste U., Jaime Garrido B., Sebastián Prado N., Carlos Navarro C. Sra. Elena Altieri L., Sr. Guillermo del Campo M. Departamento de Obstetricia y Ginecología, Unidad de Oncología Ginecológica, Clínica Las Condes. Departamento de Cirugía, Unidad de Oncología Ginecológica, Instituto Nacional del Cáncer. El Jurado acordó otorgar una "Mención Honrosa" del premio "Dr. Juan Wood Walters", año 2001, al trabajo: CINTA VAGINAL LIBRE DE TENSIÓN (CVT) MODIFICADO, ASOCIADO A CIRUGÍA VAGINAL EN EL TRATAMIENTO DE LA INCONTINENCIA DE ORINA DE ESFUERZO de los Drs. Jaime Roa B., Eutimio Roa B., Jaime Martínez N., Eduardo Osorio F., Augusto Tapia S., Gustavo Romero., Servicio de Obstetricia y Ginecología, Clínica Dávila.
4. Premio "DR. VÍCTOR MANUEL GAZITÚA GUZMÁN", año 2001, al mejor trabajo de Obstetricia, "ESPÁTULAS RECTAS PARALELAS DE VELASCO. EXPERIENCIA DEL SERVICIO CON UN NUEVO INSTRUMENTO EXTRACTOR DEL FETO EN EL PERIODO DE EXPULSIVO DETENIDO”, de los Drs. José Lattus O., Jorge Cortéz O., Ricardo San Martín T., Axel Paredes V., Gustavo Romero M., Susana Aguilera P., Hernán Rojas V., Juan Guillermo Rodríguez A., Lissette Araya A., Daniel Moretti C., Gisela Hornung C., Ghislaine Echeverria O. Servicio y Departamento de Obstetricia y Ginecología, Hospital del Salvador, Universidad de Chile. El Jurado acordó otorgar una "Mención Honrosa" del premio "Dr. Víctor Manuel Gazitúa Guzmán", año 2001, a los trabajos: "CORRELACION DEL RECUENTO DE CUERPOS LAMINARES CON EL INDICE DE LECITINA/ ESFINGOMIELINA Y/O LA PRESENCIA DE FOSFATIDILGLICEROL PARA LA DETERMINACIÓN DE MADUREZ PULMONAR FETAL", de los Drs. James Revelo R., Walter Campoverde C., Servicio de Obstetricia y Ginecología y de Neonatología, Hospital Padre Hurtado, y al trabajo "TRATAMIENTO MÉDICO CON METOTREXATO DEL EMBARAZO TUBARIO NO COMPLICADO" de los Drs. Alessandro Olivari M., Carlos Henríquez B., Gregorio Evans M., Marcelo Aguayo M., Eduardo Villanueva F., Sergio Díaz Z., Servicio y Departamento de Obstetricia y Ginecología, Hospital Clínico Regional Guillermo Grant Benavente, Universidad de Concepción.

5. Premio "DR. CARLOS MONCKEBERG BRAVO", año 2001, al mejor trabajo de Perinatología, "OCLUSION TRAQUEAL INTRALUMINAL POR VIA ENDOSCOPICA EN MODELO EXPERIMENTAL DE HERNIA DIAFRAGMATICA CONGENITA" de los Drs. Ruben A. Quinteros, Freddy Guevara-Zuloaga, Daniel Crespin, Antonieta Pedota, Patxi Aristoy, Carlos Bermúdez, Juan Carispides, Daniel Sharifker, Luis Rivera. Unidad de Perinatología del Hospital Universitario de Caracas, Grupo Venezolano de Investigación en Terapia Fetal de Caracas y Maracaibo. Instituto de Diagnostico Fetal y Terapéutico, Tampa U.S.A., Departamento de Anatomía Patológica, Hospital de Clínicas, Caracas, Facultad de Ciencias Veterinarias. El Jurado acordó otorgar una "Mención Honrosa" del premio "Dr. Carlos Monckeberg Bravo", año 2001, al trabajo "RELACION DE LOS FLUJOS CEREBRO PLACENTARIOS EN LA PREDICCION DE MAL RESULTADO PERINATAL", de los Drs. Pedro González B., Jyh Kae Nien S., 
Carlos Rondíni F., Alfredo Hernández A., Andrés Poblete L., Enrique Oyarzún E., Departamento de Obstetricia y Ginecología, Unidad de Ultrasonografía, Pontificia Universidad Católica de Chile.

La Comisión de Premios quiere dejar constancia que el Premio "Dr. Carlos Monckeberg B.", al mejor trabajo de Perinatología se otorgó en forma excepcional a un trabajo cuyos autores no son miembros de esta Sociedad, por no tener este premio reglamentación previa vigente

\section{PREMIOS AÑO 2002}

En Santiago de Chile, con fecha 25 de Noviembre 2003, a las 12:30 horas, se reúne la Comisión de Premios formada de acuerdo a los artículos 26, 27 y 29 de los Estatutos de la Sociedad Chilena de Obstetricia y Ginecología.

El Jurado constituido por el Presidente de la Sociedad, Dra. Mercedes Ruiz Flores, por el Dr. Ramón Rubio M., integrante de la Comisión de Premios y por el Secretario General Dr. Eghon Guzmán B., este último con derecho a voz solamente, acuerdan otorgar los siguientes premios correspondientes al año 2001. Cabe hacer presente que el Dr. Gabriel Leal no participó en esta reunión por su condición de salud.

1. Premio "DR. JORGE DíAZ BRAVO", año 2002, al mejor trabajo de Ingreso, "HISTERECTOMÍA VAGINAL Y CORRECCIÓN DE INCONTINENCIA URINARIA CON TVT (TENSIÓNFREE VAGINAL TAPE)" de los Drs. Rodrigo Parada C. Erasmo Bravo O. Christian Franck K., Santiago Parry R., Carlos Bennett R., Danilo Jara V., Antonio Daviu, del Servicio de Ginecología y Obstetricia, Servicio de Urología, Hospital Carlos Van Buren. El Jurado acordó otorgar una "Mención Honrosa" del premio "Dr. Jorge Díaz Bravo", año 2002, a los trabajos, "EFECTO IN VITRO DE LA INSULINA SOBRE LA CAPACIDAD CONTRÁCTIL UTERINA INDUCIDA POR OXITOCINA", de los Drs. Horacio Figueroa D., Elisa Marusic B., Magdalena González N., Francisca Barcos M., Paola Yungue V., del Servicio de Obstetricia y Ginecología, Hospital Parroquial de San Bernardo, Laboratorio de Fisiología Molecular y Celular, Universidad de Los Andes, y al trabajo "MAMOGRAFÍA FULL DIGITAL DIRECTA O EN CAMPO COMPLETO. EXPERIENCIA PRELIMINAR" de los Drs. Pilar Gazmuri P., Arturo Espinoza N., Centro de Diagnóstico Mamario y Laboratorio Citolab.
2. Premio "DR. JUAN MALFANTI", año 2002, al mejor trabajo de Casuística, "LINFOMA DE LA MAMA", de los Drs. Jaime Letzkus B., Octavio Peralta M., Galina Ivanova, Jorge Gamboa G., Alejandro Belmar, Cesar del Castillo S., Mónica Campos M., Paulina Peñaloza, Valeria Cornejo C., Eugenio Vives V. Servicio y Departamento de Obstetricia y Ginecología, Hospital Clínico San Borja Arriarán, Instituto de Radiomedicina (IRAM). El Jurado acordó también otorgar una "Mención Honrosa" del premio "Dr. Juan Malfanti", año 2002, a los trabajos, "ACATISIA INDUCIDA POR TIETILPERAZINA EN PACIENTE EMBARAZADA" de los Drs. Gabriela Vera, Aldo Varas, Servicio de Psiquiatría Hospital Félix Bulnes, Universidad de Chile, y al trabajo "MANEJO LAPAROSCOPICO CONSERVADOR DE QUISTE DERMOIDE CONTENIENDO UNA MANDIBULA Y 13 DIENTES" de los Dr. Jaime Zahri T., Juan M. Toso L., Servicio de Obstetricia y Ginecología, Hospital El Pino.

3. Premio "DR. JUAN WOOD WALTERS", año 2002, al mejor trabajo de Ginecología, "IMPORTANCIA DE LA LINFADENECTOMIA EN LA ETAPIFICACION QUIRÚRGICA DEL CANCER DE ENDOMETRIO" de los Drs. Felipe Serman V., Alejandra Díaz G., Roderick Walton L., Arturo Henríquez S., Milica Buc B., M. Elena Sánchez A., Maritza Matus I. Servicio de Obstetricia y Ginecología Hospital de Carabineros. El Jurado acordó otorgar una "Mención Honrosa" del premio "Dr. Juan Wood Walters", año 2002, a los trabajos, "RESULTADOS DE LA SALPINGONEOSTOMIA LAPAROSCOPICA EN INFERTILIDAD POR HIDROSALPINX" de los Drs. Guillermo Durruty V., Claudio Vera P., Rodrigo Macaya P., Marcelo Bianchi P., Alejandro Manzur Y., Departamento de Obstetricia y Ginecología, Unidad de Reproducción Humana, Hospital Clínico Pontificia Universidad Católica de Chile, y al trabajo "EFECTOS DE LA METFORMINA EN EL SÍNDROME DE OVARIO POLIQUISTICO ASOCIADO A INSULINO RESISTENCIA" de los Drs. Claudio Caro, Juan Fuhrer, Rodrigo Sáez, Victor Rubio, Luis Moreno y Sr. Miguel Cumsille, Servicio de Obstetricia y Ginecología Hospital San José.

4. Premio "DR. VíctoR MANUEL GAZITÚA GUZMÁN", año 2002, al mejor trabajo de Obstetricia: "MARCADORES SONOGRAFICOS DE CARDIOPATIAS CONGENITAS. DESPRO- 
PORCION DE LAS CAVIDADES CARDIACAS”, de los Drs. Fernando Viñals L., Arrigo Giuliano B. Centro AGB Ultrasonografía y Clínica Sanatorio Alemán de Concepción. El Jurado acordó otorgar una "Mención Honrosa" del premio "Dr. Víctor Manuel Gazitúa Guzmán", año 2002, a los trabajos, "COMPARACIÓN DEL INDICE LECITINA/ESFINGOMIELINA VERSUS FOSTATIDILGLICEROL EN LA EVALUACIÓN DE LA MADUREZ PULMONAR FETAL" de los Drs. Paola Pino, Enrique Oyarzún E., Rossana Vidal L., Sumie Kato C., Jorge Carvajal, Departamento de Obstetricia y Ginecología, Laboratorio de Medicina MaternoFetal, Pontificia Universidad Católica de Chile, y al trabajo "SÍNDROME ANTIFOSFOLIPIDOS Y EMBARAZO" de los Drs. Angel Correa P., Oscar Valderrama C., Raúl Angel G., Jaime Sáez C., Ernesto Villablanca O., Departamento y Servicio de Obstetricia y Ginecología, Hospital Clínico San Borja Arriarán, Universidad de Chile.

5. Premio "DR. CARLOS MONCKEBERG BRAVO", año 2002, al mejor trabajo de Perinatología, "PARTO PREMATURO ASOCIADO A COLESTASIA INTRAHEPATICA DEL EMBARAZO. ROL DEL ACIDOCOLICO EN LA MODULACIÓN DE LA VIA OCITOCINA-RECEPTOR DE OT EN EL MIOMETRIO", de los Drs. Alfredo Germain, Sumie Kato, Gloria Valdés, Jorge Carvajal, Juan Carlos Glasinovic, Departamentos de Obstetricia y Ginecología, de Nefroogía, y de Gastroenterología, Pontificia Universidad Católica de Chile.

AUSPICIO A CURSOS Y EVENTOS CIENTÍFICOS. Durante estos dos años se otorgaron auspicios y patrocinios a 20 actividades científicas tanto en Santiago, como en Provincia de gran nivel científico y que cumplían con los requisitos para ser auspiciados por nuestra Sociedad.

OBITUARIO. Dr. Bernardo Guerrero Diteaux, quien fuera miembro del Directorio de nuestra Sociedad entre los años 1978-1979 como Secretario Anual y Director en el período 1980-1981.

REVISTA CHILENA DE OBSTETRICIA Y GINECOLOGIA. Al asumir nuestra gestión fuimos informados que en Diciembre del 2001 el Laboratorio Schering, había interrumpido el convenio de patrocinio con nuestra Revista, lo que en un primer minuto parecía una situación compleja, sin embargo, tras una exitosa negociación logramos un excelente acuerdo con los laboratorios Schering, Whyet y Organon, para la impresión y distribución de la revista eliminando además una exclusividad que limitaba nuestro quehacer. El Profesor Dr. Mario Herrera Moore continuó en el cargo de Editor Jefe con gran compromiso y brillantez por lo cual le damos nuestros más fervientes agradecimientos. En total se editaron 6 números cada año de gran calidad.

Actualmente nos encontramos en los índices, Lilacs, Barcelona, Uldrich, en la Biblioteca del Congreso de los Estados Unidos, Internet y en el Proyecto Scielo, que por su importancia merece destacarse ya que es la antesala de la introducción en Medline.

REFORMA DE LOS ESTATUTOS. Durante el mes de Mayo de 2002, la Asamblea General acordó reformar los Estatutos en el artículo $N^{\circ} 5$, agregando la calidad de Socio Adherente con gran éxito en la participación de los especialistas chilenos que por diversas razones, pese a sus méritos no suelen presentar trabajos científicos de ingreso.

OTRAS ACTIVIDADES. Con el propósito de incrementar los fondos de la Sociedad, este Directorio se propuso realizar diversos Cursos y actividades científicas con gran éxito y numerosa asistencia entre los cuales se destacan los siguientes:

1. Curso "Minidosis de Estrógenos", 31 de Marzo 2002, en Santiago.

2. Curso "Tópicos en Medicina Materno Fetal", 21 y 22 de Abril de 2002, en Viña del Mar.

3. Jornadas de Obstetricia y Ginecología, 11 y 12 de Marzo de 2003, en Viña del Mar.

4. Curso "Nuevo Rol del Ginecólogo en la Salud de la Mujer ", 28 de Abril 2003, en Santiago.

PAGINA WEB. Se activo y modernizo la página Web de la Sociedad (www.sochog.cl) contratando los servicios de un equipo profesional de alta calidad liderado por el Sr. Patricio Gana. La nueva página ha funcionado a completa satisfacción del Directorio y de los Socios, siendo destacada en el ámbito científico e internacional con gran cantidad de consultas.

ASOCIMED. Sochog como miembro Fundador de Asocimed (Asociación de Sociedad Científicas Médicas), participó en todas las reuniones y actividades de esta importante asociación, siendo además elegidos como parte del Directorio para cum- 
plir el rol de Tesorero en la Directiva actual. Allí hemos participado en múltiples mesas y reuniones de trabajo interministeriales, con las Comisiones de Salud de la Cámara y del Senado, con ASOFAMECH y con otras instancias del quehacer médico del país. También se ha colaborado con la creación de algunos protocolos incluidos en las guías y pautas clínicas Ministeriales y hemos presentando nuestra evaluación de las patologías de la especialidad incluidas en el plan AUGE.

CONACEM. La Sociedad es también Socia Fundadora de la Comisión Nacional de Certificación Médica (CONACEM), siendo nuestro representante a nivel del Directorio superior el Dr. Patricio Gayán, ex Presidente de la Sociedad, y en la Comisión de Gineco-Obstetricia el Dr. Gustavo Gormaz, también ex Presidente de SOCHOG.

MINISTERIO DE SALUD. Por Decreto del Ministerio de Salud se creo una Comisión permanente Minsal-Sochog, integrada por los Drs. René Castro, Jefe del Programa de la Mujer de MINSAL, el Sr. Pablo Venegas, Jefe de la División Económica y Administrativa del Ministerio y por SOCHOG la Dra. Mercedes Ruiz, Presidente, y el Dr. Patricio Silva, Tesorero. Esa comisión sesionó mensualmente durante los años 2002 y 2003, para coordinar, planificar y evaluar todas las actividades relacionadas con el Congreso Mundial FIGO 2003.

OTRAS ACTIVIDADES. Participación activa de diversos miembros del Directorio y de la Sociedad en los medios de comunicación a lo largo de toda nuestra gestión, con el propósito de dar a conocer importantes tópicos de la especialidad y muy especialmente promover FIGO 2003.

FIGO (EXCUTIVE BOARD). En Junio del 2002, Chile participó en Estambul, Turquía, como miembro integrante de los 24 países que conforman el Executive Board de FIGO (verdadero Senado que define las políticas y planes de la Federación), donde entre otras importantes decisiones se constituyó el Comité Organizador definitivo del Congreso Mundial correspondiéndole a quien les habla la calidad de Co-Presidente del mismo. Allí se consiguió además que la próxima reunión del Executive Board se realizará en Chile, siendo la primera vez que ello sucedía en Latinoamérica. Esa reunión se efectuó en Junio de 2003 con gran éxito y la participación de todos los países integrantes, quienes consideraron esta oportunidad como una an- tesala del Congreso Mundial que se realizaría en Noviembre de 2003.

Un nuevo encuentro se produjo en Enero de 2004 en Londres, con el propósito de conocer a los nuevos integrantes, tanto officers, como miembros del Executive Board, que fueron elegidos en la Asamblea General del día 7 de Noviembre de 2003, en Santiago de Chile, y en la cual asumió como Presidente el Dr. Arnaldo Acosta. Chile continuará perteneciendo a esta instancia hasta el año 2006, siendo necesario iniciar un intenso lobby para la reelección de nuestro país durante la Asamblea General del Congreso Mundial de Malasia FIGO 2006.

FLASOG 2002. En el mes de Octubre se realizó en Santa Cruz de la Sierra, Bolivia, el Congreso de la Federación Latinoamérica de Obstetricia y Ginecología (FLASOG), que además conmemoraba las bodas de oro de la Federación y en el cual Chile participó con una importante delegación de más de 40 médicos y 25 conferencistas. Tuvimos también el agrado que 2 distinguidos miembros de nuestra Sociedad, la Dra. Eneida Aguilera y el Dr. Rodolfo Wild fueran declarados "Maestro Latinoamericano de la Especialidad". Chile presentó un hermoso stand para promocionar FIGO 2003 y se nos otorgó una sesión plenaria para exponer ante la audiencia el evento e invitarles a participar. Allí anunciamos la creación del fondo SOCHOG que con la participación de la industria farmacéutica mundial y la colaboración de Events International Meeting Planners, nos permitió bonificar a dos mil médicos latinoamericanos con inscripciones preferenciales a precios rebajados para el Congreso Mundial FIGO 2003.

COMITÉ DE DERECHOS SEXUALES $Y$ REPRODUCTIVOS DE FLASOG. Chile tuvo una activa participación en ese Comité a través de la Dra. Patricia Aliaga, quien cumplió a cabalidad con las expectativas del Directorio.

COMITÉ DE MORTALIDAD MATERNA DE FLASOG. En Noviembre de 2003, se creó el Comité de Mortalidad Materna de Flasog, realizándose una primera reunión de coordinación en conjunto con OPS en ciudad de Guatemala, en Marzo del 2004. Durante ese encuentro se analizaron las cifras e indicadores materno infantiles de los distintos países latinoamericanos siendo Chile uno de lo mejor evaluados en todos los parámetros, por lo que recibimos numerosas felicitaciones de los participantes. 


\section{CONGRESO MUNDIAL FIGO 2003}

Como es de todos conocido nuestro país obtuvo la sede del XVII Congreso Mundial FIGO 2003, en el mes de Agosto de 1997, en Copenhage, durante la asamblea general del XV Congreso de la Federación Internacional de Ginecología y Obstetricia. A partir de ese momento y en sucesivos directorios se inició una ardua tarea no excenta de múltiples inconvenientes y dificultades que culminó en noviembre de 2003 con un éxito extraordinario en todos los ámbitos. Al punto de ser considerado por FIGO como el más exitoso de cuantos Congresos se hayan realizado hasta el momento.

Entre los principales hitos deseo destacar el extraordinario aporte del Gobierno de Chile, quién a través del Ministerio de Salud, firmó un convenio en Mayo del 2002 con la Sociedad Chilena de Obstetricia y Ginecología, respaldado por las Comisiones de Salud de la Cámara y del Senado y aprobado por la Contraloría General de la República que definió los pasos a seguir para la culminación de un evento de esta importancia. Como ya he mencionado anteriormente la creación del Comité de trabajo MINSAL - SOCHOG con reuniones mensuales monitoreó a lo largo de estos 2 años la evolución del proceso entregando un informe final minucioso a la autoridad competente.

El Directorio de la Sociedad en pleno se comprometió en forma irrestricta y respaldó las acciones emprendidas tanto a nivel nacional como internacional. Con gran satisfacción manifiesto que el respaldo entregado a mi persona hizo posible el enorme esfuerzo y sacrificio que esta titánica tarea me demandó. Fue necesario realizar innumerables viajes con largos desplazamientos que a veces sólo duraban horas de permanencia en los diferentes sitios para promocionar a nuestro país y en tal sentido deseo hacer una mención muy especial al Secretario General de la Sociedad Dr. Eghon Guzmán, quien me acompañó en muchas oportunidades, colaborando en forma significativa al éxito de está empresa.

Deseo también destacar la colaboración de la Industria Farmacéutica Nacional quien nos permitió editar 3 números de la Revista Rumbo al Mundial la cual se convirtió en una excelente vitrina promocional a lo largo del país.

RESULTADOS DEL CONGRESO MUNDIAL. Participaron 8.400 delegados de 137 países. De ellos 2.000 latinoamericanos y alrededor de 1.050 profesionales chilenos. A lo anterior se suma 1.073 acompañantes y aproximadamente 2.000 representantes de la industria farmacéutica internacional y casas de electromedicina, que en su conjunto representa alrededor de 11.500 personas como total de participantes. Ello constituye un récord en número y en cantidad de países representados en los Congresos FIGO.

El balance económico para el país fue óptimo lo que se reflejó en la total ocupación de la hotelería nacional, de todos los destinos turísticos ofrecidos y del incremento notable en las ventas del comercio, dado lo cual existe gran satisfacción en los Ministerios de Hacienda y Economía por estos excelentes resultados.

Desde el punto de vista científico contamos con 465 conferencistas internacionales en los más diversos tópicos de nuestra especialidad y con profunda satisfacción deseo destacar la importante participación de docentes nacionales incluida una de las 6 conferencias claves del Congreso dictada por el Dr. Horacio Croxatto y en la cual tuve el honor de ser la Presidenta de esa sesión. Además hubo 77 contribuciones chilenas de gran nivel y publicadas en el libro de resúmenes.

PROGRAMA SOCIAL. El acto inaugural en el frontis del Museo de Bellas Artes con la participación de su excelencia, el Presidente de la República, las más altas autoridades del país y la actuación extraordinaria de la distinguida soprano Cristina Gallardo-Dhomas acompañada de la orquesta sinfónica de Santiago, será sin duda un recuerdo inolvidable que nos acompañará para siempre.

El carnaval chileno realizado en el Club Hípico y donde la principal atracción fue el clásico FIGO ganada por el caballo SOCHOG, fue una expresión de nuestra costumbres y artesanías nacionales que deleitaron a todos los participantes. Los actos culturales del día 4 de noviembre, función de ópera de gala en el Teatro Municipal y concierto en la iglesia de los Sacramentinos, pusieron de manifiesto la calidad de nuestros artistas internacionales en el campo de la lírica y de la música, ya que ambas funciones fueron enteramente con artistas nacionales.

Finalmente la cena de clausura en una de las más importantes viñas del país y a la cual acudieron más de 2.000 personas, puso el broche de oro al programa social. Deseo destacar que cada uno de los asistentes a esta cena final recibió como regalo de SOCHOG una botella de vino con el logo del Congreso y de la Sociedad. 
PREMIOS OTORGADOS DURANTE FIGO 2003 A MEDICOS CHILENOS

El Dr. Aníbal Faúndez, recibió el premio por trabajos distinguidos durante la inauguración del día 2 de noviembre y la Dra. Mercedes Ruíz Flores recibió el premio a la Mujer Ginecóloga, destacada a nivel Mundial durante la asamblea general realizada el día 7 de noviembre, en Santiago.

FELICITACIONES RECIBIDAS. La Sociedad Chilena de Obstetricia y Ginecología, recibió numerosos correos electrónicos, faxes y diversas manifestaciones de felicitación, tanto nacionales como internacionales, incluyendo los elogios de la Presidencia de la República y del Ministerio de Salud. Todos estos testimonios se encuentran en los archivos de la Sociedad y de ellos nos sentimos muy orgullosos.

TAREAS PENDIENTES. Entre las tareas pendientes cabe destacar: 1) Edición y publicación de un libro denominado "Historia del Congreso Mundial FIGO 2003 en Chile. 2) Mantener la posición de liderazgo de SOCHOG tanto en el Executive Board, como en la posibilidad de obtener un cargo de officers en el Directorio de la Federación Internacional.

AGRADECIMIENTOS. No puedo finalizar esta cuenta sin mencionar nuevamente los agradecimientos al Directorio de SOCHOG que me acompañó en mi gestión, al Gobierno de Chile por su ayuda invaluable muy especialmente a su Excelencia el Presidente de la República, Don Ricardo Lagos, por su brillante y emotivo discurso en la ceremonia inaugural, a la Alcaldía de Santiago, por la colaboración permanente tanto en la facilitación de los espacios públicos, como del personal técnico competente en desplazamientos en reuniones masivas, al Senado de la República y a la Cámara de Diputados por la aprobación de las iniciativas legales facilitadoras del Congreso, al Colegio Médico y a las Universidades, dispuestas en todo momento a participar con nosotros, y a Events International Meeting Planners de Canadá y su filial chilena Events Chile, por su gran profesionalismo y responsabilidad como organizador de eventos de esta magnitud que permitió el lucimiento de nuestro país en todos sus aspectos.

El equipo de secretariado merece una mención especial por su esfuerzo, responsabilidad, lealtad y eficiencia, dirigidos por la Sra. Ingrid Stevens, colaboraron en forma sustancial a mi labor durante este período 2002-2003 y muy especialmente a la realización del Congreso Mundial FIGO 2003.

Al despedirme quisiera sintetizar mi labor en los siguientes términos: entregamos una Sociedad ordenada en lo administrativo, solvente en lo económico, respetada internacionalmente en lo científico y sobretodo con buenas relaciones entre todos sus miembros, es decir en paz. Muchas gracias.

Dra. Mercedes Ruiz Flores 